\title{
A survey on 564 cases of inpatients with anxiety and depression in general hospitals in 2015
}

\author{
Yulian Jiang, Dong Wang, Xiulian Wen, Tingting Li, Ruidong Jing, Yongdong Li, Weidong Guo, Hong Cui, Ziling Li \\ The Third Affiliated Hospital of Inner Mongolia Medical University, Baotou, Inner Mongolia, China
}

Received: January 13, 2018

DOI: $10.14725 /$ dcc.v5n1p19

\author{
Accepted: February 20, $2018 \quad$ Online Published: March 10, 2018 \\ URL: http://dx.doi.org/10.14725/dcc.v5n1p19
}

\begin{abstract}
Objective: To understand the prevalence of anxiety and depression in medical patients in general hospitals and find out main influencing factors.

Methods: According to the inclusion criteria, a total of 564 inpatients from the Third Affiliated Hospital of Inner Mongolia Medical University were selected during January to June of 2015. Patients were assessed for anxiety and/or depression by use of Hospital Anxiety and Depression Scale (HADS), Hamilton Anxiety Scale (HAMAS) and Hamilton Depression Scale (HAMDS). Meanwhile, final diagnosis was made on the basis of diagnostic criteria listed in Diagnostic and Statistical Manual of Mental Disorders - Fourth Edition (DSM-IV).

Results: In different departments, the positive rate (HADS $\geq 8$ ) was the highest in Department of Oncology (45.42\%), and the lowest in Department of Gastroenterology (16.05\%). After assessment with the help of HAMD and HAMA, the prevalence of anxiety/depression was the highest in Department of Oncology (46.43\%), and the lowest in Department of Gastroenterology $(16.05 \%)$. There was no statistical significance in diagnostic results acquired from HAMD and HAMA $(p=.071)$. Two types of diagnostic methods were highly consistent $(\kappa=0.852, p=.000)$. Inpatients aged from 40 to 59 years, with junior high school education or below, very tiring work, poor marital status (separated, divorced, widowed), unharmonious family, low personal income, completely self-paying and family history of anxiety and depression, currently unable to take care of themselves were more prone to anxiety and depression $(p<.05)$.

Conclusions: The prevalence of anxiety and depression in medical patients in general hospitals is high due to many influencing factors. It is necessary to establish an effective diagnosis and treatment system for anxiety and depression, in order to make patients easy to receive an early and comprehensive treatment and improve their life quality.
\end{abstract}

Key Words: Depression, Anxiety, Mental health, General hospital, Inpatients

In different regions of China, the reported prevalence of anxiety and/or depression in general hospitals differs, ${ }^{[1]}$ however, so far, there still lacks epidemiological studies on a large number of samples. In some similar domestic surveys, patients mostly came from a certain specialty or suffered from certain specific diseases, ${ }^{[2-4]}$ i.e., the study was mainly on the basis of a specific disease. In addition, length of stay for selected cases varies, thereby, such studies can not really represent the prevalence of anxiety and/or depression in patients in general hospitals. In recent years, the incidence of cancer has been increasing year by year, and the prevalence of anxiety and/or depression in cancer patients is also very high. However, investigations and studies on the comparison of dysthymic disorder between Internal Medicine Departments and Department of Oncology are rarely reported at present. ${ }^{[5,6]}$ Inner Mongolia Autonomous Region is an

\footnotetext{
*Correspondence: Yulian Jiang; E-mail: dcc68@ncspress.com; Address: The Third Affiliated Hospital of Inner Mongolia Medical University, Baotou, Inner Mongolia, China.
} 
area inhabited by ethnic groups, and it is an economically undeveloped area. The prevalence of anxiety and/or depression may be lower than that in the developed areas. However, there have been no reports on surveys and researches about anxiety and depression in general hospitals in Inner Mongolia. This study is intended to investigate the prevalence of anxiety and/or depression in Internal Medicine Departments (Department of Gastroenterology, Department of Cardiology, Department of Neurology) and Department of Oncology in general hospitals and analyze the influencing factors.

\section{Objects and methods}

\subsection{Research objects}

A total of 564 inpatients from Internal Medicine Departments (Department of Neurology, Department of Gastroenterology, Department of Cardiology, Department of Endocrinology and Department of Oncology) were chosen from the Third Affiliated Hospital of Inner Mongolia Medical University during January to June of 2015. Enrollment criteria were as follows: Patients with age $\geq 18$ years, sex unlimited; primary school education and above or sufficient audio-visual and expression ability to complete the scale; patients with clear consciousness, able to answer questions clearly and independently; patients' conditions allow for investigation and communication without obvious difficulties; all patients hospitalized in the selected departments during the survey; patients with the hospital stay of 1 to 4 weeks (In 2014, the average length of stay for patients in each department was 7 to 22 days); patients who agreed to be enrolled with informed consent forms signed. Exclusion criteria were as follows: inpatients with non-medical purposes; patients with consciousness disorders; patients with severe cognitive disorders and aphasia; patients who were unable to complete the research due to severe mental or physical dysfunction; patients with hyperthyroidism or hypothyroidism; patients who were taking hormones or spirit drugs; patients who had been screened in other departments; patients who were ultimately diagnosed with no diseases related to the above departments. As to patients who were informed and screened but refused to receive diagnostic examinations performed by psychiatrists, they were treated as loss of follow up.

\subsection{Contents and methods of investigation}

After inpatients who met the enrollment criteria signed informed consent forms, the investigators who had undergone a unified and professional training would fill in HADS questionnaires and self-established questionnaires for general information, and consider patients with HADS $\geq 8$ as anxietyand/or depression-positive patients. ${ }^{[7]}$ According to Diagnostic and Statistical Manual of Mental Disorders - Fourth 20
Edition (DSM-IV), two doctors who were trained strictly and occupied in psychological medicine applied Hamilton Anxiety Scale (HAMAS) and Hamilton Depression Scale (HAMDS) to anxiety and/or depression assessment performed to all patients, and made final diagnosis on the basis of diagnostic criteria listed in DSM-IV. ${ }^{[8]}$

\subsection{Statistical analysis}

SPSS13.0 software was applied to statistical analysis, and chi-square test was used to make analysis of patients with anxiety/depression disorders in different departments. $\kappa$ coefficient was used to evaluate the consistency of HADS with HAMAS and HAMDS methods. Non-conditional logistic regression analysis, with dichotomous dependent variables applied, was used to analyze the influencing factors for anxiety and depression in medical inpatients in general hospitals, and the difference $p<.05$ was statistically significant.

\section{Results}

\subsection{HADS assessment on the prevalence of anx- iety/depression disorders in different depart- ments}

HADS was used to determine the state of anxiety/depressive disorders in research objects, the number of whom with HADS $\geq 8$ was 146 in total, and the prevalence of anxiety/depression disorders was $25.89 \%$ (see Table 1). The positive rate of anxiety/depression disorders in Department of Neurology was $22.95 \%$, and $16.05 \%$ in Department of Gastroenterology, 24.74\% in Department of Endocrinology, $24.37 \%$ in Department of Cardiology and $45.42 \%$ in Department of Oncology. The difference among different departments was statistically significant $\left(\chi^{2}=21.513, p=.000\right)$, with the highest positive rate in Department of Oncology and the lowest in Department of Gastroenterology.

\subsection{HAMD and HAMA assessments on the preva- lence of anxiety/depression disorders in differ- ent departments}

HAMD and HAMA were used by psychiatrists to make a diagnostic assessment to research objects. The number of patients with anxiety/depression was 135 , and the prevalence was $23.94 \%$ (see Table 2). The prevalence of anxiety/depression disorders in Department of Neurology was $19.13 \%$, and $16.05 \%$ in Department of Gastroenterology, $20.62 \%$ in Department of Endocrinology, 23.53\% in Department of Cardiology and $46.43 \%$ in Department of Oncology. The difference among different departments was statistically significant $\left(\chi^{2}=29.031, p=.000\right)$, with the highest prevalence in Department of Oncology and the lowest in Department of Gastroenterology.

ISSN 2375-8449 E-ISSN 2375-8473 
Table 1: HADS assessment on the positive rates of anxiety/depression disorders in different departments

\begin{tabular}{llll}
\hline Department & Investigated Cases (n) & Positive Cases (n) & Positive Rate (\%) \\
\hline Department of Neurology & 183 & 42 & 22.95 \\
Department of Gastroenterology & 81 & 13 & 16.05 \\
Department of Endocrinology & 97 & 24 & 24.74 \\
Department of Cardiology & 119 & 29 & 24.37 \\
Department of Oncology & 84 & 38 & 45.24 \\
Total & 564 & 146 & 25.89 \\
\hline
\end{tabular}

Table 2: HAMD and HAMA assessments on the prevalence of anxiety/depression disorders in different departments

\begin{tabular}{llll}
\hline Department & Investigated Cases (n) & Positive Cases (n) & Positive Rate (\%) \\
\hline Department of Neurology & 183 & 35 & 19.13 \\
Department of Gastroenterology & 81 & 13 & 16.05 \\
Department of Endocrinology & 97 & 20 & 20.62 \\
Department of Cardiology & 119 & 28 & 23.53 \\
Department of Oncology & 84 & 39 & 46.43 \\
Total & 564 & 135 & 23.94 \\
\hline
\end{tabular}

2.3 The consistency of HAMD and HAMA assessments with HADS assessment on anxiety/depression

McNemar test showed that the difference between the two diagnostic results was of no statistical significance $(p=$ .071). According to $\kappa$ test, the consistency coefficient of the two diagnosis methods was $\kappa=0.852$, indicating that the two diagnostic methods were highly consistent (see Table $3)$.

Table 3: The consistency of HAMD and HAMA assessments with HADS assessment on the positive rate of anxiety/depression

\begin{tabular}{llll}
\hline \multirow{2}{*}{ HADS Assessment } & \multicolumn{2}{l}{ HAMD and HAMA Assessments } & \multirow{2}{*}{ Total } \\
\cline { 2 - 3 } & With illness & Without illness & \\
\hline Positive & 125 & 21 & 146 \\
Negative & 10 & 390 & 400 \\
Total & 135 & 411 & 546 \\
\hline
\end{tabular}

\subsection{Relative influencing factors for anxiety and de- pression}

Inpatients aged from 40 to 59 years, with junior high school education or below, very tiring work, poor marital status (separated, divorced, widowed), unharmonious family, low personal income, completely self-paying and family history of anxiety and depression, currently unable to take care of themselves were more prone to anxiety and depression (see Table 4).

\section{Results}

With the change of the medical model, the phenomenon of physical diseases accompanied by anxiety and depression has aroused wide attention in the medical community and the patients themselves. However, in recent years, clinicians in general hospitals have neglected the knowledge and treatment of anxiety and depression in different degrees. Additionally, people in our country were born with inherently conservative views and values, making the prevalence of anxiety and depression continue to rise, but the recognition rate as well as diagnosis and treatment rate is obviously low. ${ }^{[3]}$

\subsection{Prevalence of anxiety and depression in differ- ent departments}

HADS is a self-rating scale designed to investigate whether patients with physical diseases in general hospitals suffer from anxiety and depression. It is easy to operate and widely used in mental health surveys and researches conducted in general hospitals and primary health care institutions. ${ }^{[1]}$ This study showed that nearly one fourth of inpatients in general hospitals suffered from anxiety or depression, and the prevalence was significantly higher than that in the general population. This study was designed for medical inpatients. Patients in Internal Medicine Departments mainly suffered from chronic diseases (such as cardiovascular and cerebrovascular diseases, diabetes and tumors), with long course of disease and easily recurrent seizures. Some patients even need a lifelong treatment, so they could be more prone to mental disorders. ${ }^{[9]}$ The prevalence of anxiety and depression in Department of Oncology was signif- 
icantly higher than that in the other surveyed departments, followed by Department of Cardiology, Department of Endocrinology, Department of Neurology and Department of
Gastroenterology. Nearly one-fifth of patients were diagnosed with anxiety/depression by psychiatrists.

Table 4: Univariate logistic regression analysis

\begin{tabular}{lllllll}
\hline Influencing Factors & $\boldsymbol{\beta}$ & $\mathbf{S E}$ & Wald & $\boldsymbol{p}$ & OR & OR $(\mathbf{9 5 \%} \mathbf{C I})$ \\
\hline Age (40-59 years) & 1.05 & 0.27 & 14.82 & .00 & 2.87 & $(1.68,4.91)$ \\
Junior high school education or below & 0.51 & 0.19 & 7.14 & .01 & 1.67 & $(1.15,2.42)$ \\
Very tiring work & 2.43 & 0.61 & 15.92 & $<.0001$ & 11.31 & $(3.44,37.25)$ \\
Poor marital status (separated, divorced, widowed) & 0.92 & 0.13 & 50.33 & $<.0001$ & 2.51 & $(1.95,3.24)$ \\
Unharmonious family & 0.20 & 0.08 & 5.78 & .02 & 1.22 & $(1.04,1.43)$ \\
Low personal income & 2.25 & 0.62 & 13.19 & .00 & 9.48 & $(2.82,31.89)$ \\
Completely self-paying & 0.63 & 0.24 & 6.77 & .01 & 1.88 & $(1.17,3.03)$ \\
Family history of anxiety & 1.70 & 0.36 & 22.25 & $<.0001$ & 5.49 & $(2.71,11.14)$ \\
Family history of depression & 0.87 & 0.24 & 12.70 & .00 & 2.38 & $(1.48,3.84)$ \\
Poor health condition (currently unable to take care of themselves) & 0.43 & 0.10 & 19.68 & $<.0001$ & 1.54 & $(1.27,1.86)$ \\
\hline
\end{tabular}

\subsection{Relative influencing factors for anxiety and de- pression}

This study has shown that, from the perspective of age, middle-aged patients are more prone to anxiety and depression disorders. It is considered to be related to mental stress, as well as social, work and family burdens. The family history of anxiety and depression, a liability factor, is also associated with the occurrence of anxiety and depression. It is considered to be related to heredity effects, poor treatment compliance and drug withdrawal. Patients with poor health and unable to take care of themselves are more prone to anxiety and depression. As the severity of physical diseases deepens, the course of disease is prolonged; accordingly, patients' psychological endurance becomes worse, eventually leading to the occurrence and exacerbation of anxiety and depression.

However, the limitation of this study is that only one GradeIII A hospital in Inner Mongolia has been investigated. The investigated department is limited to 5 departments, in- cluding Department of Neurology, Department of Cardiology, Department of Gastroenterology, Department of Endocrinology and Department of Oncology. Therefore, the source of samples and its characteristics should be taken into account to draw a conclusion in this study.

In summary, the incidence of anxiety/depression disorders is high in medical inpatients in general hospitals, and affected by age, social and psychological factors etc. Therefore, it is imperative to establish an effective diagnosis and treatment system for anxiety and depression with doctors from different departments involved, and arouse the importance and recognition of anxiety/depression in non-psychiatrists in general hospitals, so that patients can be ensured to receive an early and comprehensive treatment and improve their life quality.

\section{Conflicts of Interest Disclosure}

The authors have no conflicts of interest related to this article.

[5] Zhang YX, Zhang HQ, Zhang J. Survey on the state of anxiety and depression in patients hospitalized in internal medicine departments in general hospitals. China Practical Medical. 2013; 18(8): 256-257.

[6] Ye RF, Geng QS, Ou LM, et al. Correlative analysis between anxiety, depression and somatic symptoms in outpatients in Department of Cardiology. South China Journal of Cardiovascular Diseases. 2013; 19(1): 48-51.

[7] Xia GZ, Zhang ZZ. The correlation between sleep quality and anxiety on 167 patients with migraine. Chinese Journal of Medical Guide. 2014; 16(4): 616-617.

[8] Heffner CL. Diagnostic and statistical manual of mental disorders (DSM). Washington, DC: American Psychiatric Association; 1994. 143-147 p.

[9] Zhao M, Yu GL, Yang TL. Investigate the Incidence of Anxiety and Depression in Outpatients from Cardiovascular Department in a General Hospital. Chinese Journal of Clinical Psychology. 2012; 20(2): 184-189. 